\title{
Business/IT Alignment in Practice: Lessons Learned from a Requirements Project at P\&G
}

\author{
Gil Regev ${ }^{1}$, Julien Favre ${ }^{2}$, Erich Hayek ${ }^{2}$, Paul Wilson ${ }^{2}$, and Alain Wegmann ${ }^{1}$ \\ ${ }^{1}$ Ecole Polytechnique Fédérale de Lausanne (EPFL), \\ School of Computer and Communication Sciences \\ CH-1015 Lausanne, Switzerland \\ \{gil.regev, alain.wegmann\}@epfl.ch \\ ${ }^{2}$ Procter and Gamble Information Technology (Information and Decision Solutions, IDS) \\ \{favre.j, hayek.e,wilson.pa\}@pg.com
}

\begin{abstract}
One of the main objectives of the alignment of Business and IT in both small and large organizations is to improve the chances for an IT department to provide services that result in business success. An essential phase for this alignment is the definition of business requirements. In this paper we describe the successful use of a blend of industrial and academic requirements methods for an IT system development at Procter and Gamble, (P\&G), UK. We identify two main elements for the success of this phase: (1) the combination of methods from $P \& G$ and academia, (2) the focus of $P \& G$ 's IT department on providing business solutions. This shift is accompanied by a move from writing specifications of IT systems towards defining business requirements. We discuss how these elements explain the success of this phase and propose research directions that may ease the introduction and use of requirements definition methods in organizations.
\end{abstract}

\section{Introduction}

Business and IT alignment is often considered to be a necessary ingredient for improving the chances of success in small and large organizations [2, 3], even those that do not produce IT products or services. In a recent study about enablers and inhibitors of business and IT Alignment [5], the following aspects were reported as the two main enablers: "IT understanding of the firm's business environment" and "Close partnership between IT and business." The "Lack of effective business communication with IT" scored third in the list of inhibitors, just after "Lack of senior executive support for IT" and "Lack of influence of headquarters' leadership." These enablers and inhibitors are addressed in a large part during the definition of business requirements. Furthermore, IT people need to improve their understanding of the business [3]. The question of business and IT collaboration through Requirements Engineering is therefore becoming an important element in Business and IT alignment. In this paper we describe the successful use of a blend of industrial and academic requirements methods for an IT system development at Procter \& Gamble (P\&G). The project resulted in improvements in both $P \& G$ and academia ${ }^{1}$.

\footnotetext{
${ }^{1}$ A shorter version of this paper was published as a technical report, see [9].
} 
The project was performed as a 6 months long Master's project by Julien Favre in 2005. It was deemed a success by $P \& G$ as well as from the academic standpoint. For $\mathrm{P} \& \mathrm{G}$, the project established a firm set of requirements and prototypes that is now being used by the development team.

For the academic side, the adoption process described by Moore [7] was used to improve the SEAM Enterprise Architecture method [11, 12], and integrated in an Enterprise Architecture course given at EPFL [10].

The paper is an experience report and only relates the opinions of its authors. No systematic research or formal validation has been carried out.

In Section 2 we present the project on which this paper is based. In Section 3 we present the RE methods that were used in this project. In Section 4 we explain the influence that this project had on academia. In Section 5 we discuss the key learning points we draw from this project before concluding in Section 6.

\section{The Project}

Procter \& Gamble $(\mathrm{P} \& \mathrm{G})$ is one of the leading global manufacturers of branded consumer goods. P\&G's IT department has changed its mission some years ago from developing IT systems to providing business solutions. This shift is reflected in the name change from IT to IDS (Information and Decision Solutions). IDS is part of the internal business unit Global Business Services (GBS). GBS provides business process services and business process transformation to all other business units. IDS focuses on service creation and service management while operations and transactional processes are mostly outsourced. IDS acts as mediator between the business-related organizational units and the service vendors (see Figure 1). Business transformation is enabled by IDS in three main ways: (i) analysis of the needs (i.e. the requirements) expressed by the business organizational units, (ii) the technology and vendor selection, (iii) set up of end-to-end services. The IDS contribution is evaluated in terms of its impact on the P\&G Brand's "in-market" performance.

$P \& G$ worked hard on managing the innovation and growth process, while remaining focused on keeping financial performance in the top third of the industry peer group every year. This is done via very vigorous tracking of how many of P\&Gs ideas get prototyped, how many of the prototypes get developed and qualified, and how many of those get test marketed and then successfully commercialized. Procter \& Gamble has taken its success rate, which in 1999 was about 15 percent or 20 percent to above 60 percent today.

$P \& G$ uses the concept of "initiative" for the development of any new product, a change of an existing product or in general any project that implies involvement of resources or capital across the organization.

Consequently, the initiative Tracking and initiative Management Optimization has become one of the key focus areas of IDS. The opportunity and goal is to create high value for $\mathrm{P} \& \mathrm{G}$ by improving initiative success and reducing initiative development costs and time.

The management of initiatives today is a complex task due to the large number of organizational units, functions and IT systems involved (e.g. R\&D, Supply Chain, Sales and Marketing Analysis) and the difficulty to obtain the necessary data to monitor an initiative's progress. 


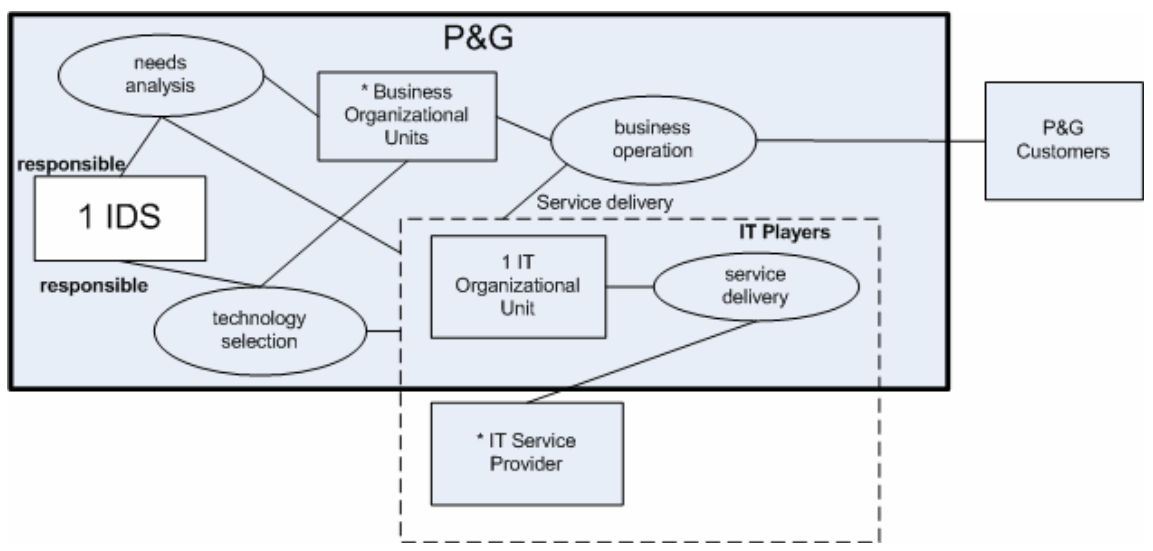

Fig. 1. $P \& G$ IT project organization

The project on which this paper is based was aimed at defining the requirements for an integrated IT solution for the part of the initiative that monitors the success of the initiative once it was launched.

\section{The RE Methods Used}

At the initial stage of the project, it was decided to use Adoption Methodology because it is the recommended approach inside P\&G. The use of Adoption Methodology was supplemented by the use of Contextual Inquiry [1,4] and SEAM goal and belief models [8]. Contextual Inquiry improved the collection of information. SEAM goal and belief models provided an early requirements method, increased the focus on certain elements such as relationships between stakeholders and improved some aspects of Adoption Methodology.

\subsection{Adoption Methodology}

The goal of Adoption Methodology is to improve technology adoption, and hence project success rates. It has the secondary benefit of ensuring IT project leaders understand the business implications of their technology.

The need for focusing on adoption came with the change of the definition of success for an IT project. The definition of success evolved from the roll out of a product to the actual adoption of the product by its intended users. This change triggered the need for developing a standard approach, that is, Adoption Methodology. Technology adoption is a term now used broadly within IDS. It is widely understood that adoption should be the end goal of any IT project. By leveraging Adoption Methodology, IT project managers can now set realistic technology adoption measures, and take specific steps to deliver on them.

Adoption Methodology is based on Moore's method for marketing high-tech products to mainstream customers [7]. It was initially developed in 2002 to increase adoption rates of Procter \& Gamble IT projects in North America. Instead of targeting customers in a market segment, as is the case in Moore's method, Adoption 
methodology targets Procter \& Gamble employees who are users of the IT systems developed by IDS (IDS customers). Hence, when we refer to users in the rest of the paper, we mean IT system users, not users of Procter \& Gamble products.

Adoption Methodology blends IT industry best practices, user psychology, macroeconomics, specific training techniques, market segmentation methods, and business understanding to create an optimal training and deployment plan for any IT project. Adoption Methodology provides a way to translate the business understanding into a business validated IDS solution. Adoption Methodology uses the concepts of segmentation, scenarios and whole product.

The segmentation is the grouping the potential users of the system by their way of using it. For example, if some users will use a subset of the capabilities of the system and other users will use another distinct subset, they will form two segments. A segment is a group of potential users that will benefit from the same set of opportunities.

Scenarios represent a way of communicating an IT system functionality to a particular segment of users. There is one scenario for each opportunity and segment. The scenarios are also used to assess their value and probability of being adopted.

A whole product is the combination all the aspects that users expect, mostly implicitly from a new IT system. It includes among other things the IT system itself, the IT service associated with the system and the training.

Adoption Methodology is strongly "value focused" and helps to understand where the value of a product comes from. It gives a very realistic view on which functionality provides which value to which user. For example, the value and users' benefits can be detailed by segment and consequently can be more accurate than if all users are treated in the same way. In effect, an overall value or overall benefit is not very useful since it is unlikely that it will be valid for all users.

Adoption Methodology addresses several steps in an IT project: the business understanding, the complete requirements (including changes to the business processes), the system rollout, the users training and the marketing of the solution. The concrete adaptation of Adoption Methodology to this project involved the following steps:

1. Early requirements - business understanding

2. Initial users involvement

3. 1st round of interviews - business understanding

4. Scenarios development

5. 2nd round of interviews - scenario assessment

The first round of interviews is structured so as to uncover users' pain areas that are then converted into opportunities through the use of scenarios.

In its simplest form, the Adoption Methodology addresses nine core questions:

1. Who is the target customer?

2. What is this customer's compelling reason to use the envisioned solution?

3. What is the whole product solution that fulfills this reason?

4. Who are the key partners and allies who might be part of this whole product?

5. What is the optimal deployment method or scheme (e.g. online, face to face)?

6. What is the customers' cost of using the envisioned solution?

7. Who, what, and where are there competing solutions?

8. What is the optimal positioning of the envisioned solution?

9. Who is the next target? 
These questions are asked for each functionality. Going through these questions leads to:

- A clear vision, or goal, articulating the project's desired end state (what work will be done differently, and by whom).

- A clear action plan that optimizes available resources and delivers on the vision or goal.

In the second round of interviews, we made several iterations. We started with a very simple set of requirements and a very simple mock-up.

Then, during each interview we improved the mock-up and got more and more details about the requirements. In order to validate the addition of the requirements, we systematically asked two questions:

- Why do you need this requirement?

- Which precise actions can you take with this functionality?

The 9 questions of the adoption methodology and these 2 questions have two distinct uses. The two questions provided us a good and rigorous way of selecting the more interesting functionalities. To assess the value of those selected functionalities and their chances of being adopted, we used the 9 questions listed above.

They were also the base for defining the compelling reasons to buy and the value of a given functionality.

\subsection{Contextual Inquiry}

Contextual Inquiry [1, 4] is an interviewing technique where interviewers establish an apprentice-master relationship with the interviewees. The interviewer plays the apprentice role and the interviewee the master role. The interview is done on the working premises of the interviewee and while the interviewee accomplishes the normal tasks that the interviewer wants to learn about. Contextual Inquiry is based on the assumption that during normal interviews interviewees talk in generalities and have difficulties in remembering and expressing the details that the interviewer needs in order to define accurate requirements. It is often difficult to introduce Contextual Inquiry in practice because it takes more time than traditional interviews and because of the interference with normal operations.

For the interviews, we used some of the guidelines from Contextual Inquiry. The main constraint was the time in our case; consequently, we haven't been able to use every aspect of this method.

As a general principle, we tried to optimize the time we spent with system's users and sometimes this optimization was not compatible with the contextual inquiry principles.

On the other hand, we maximized the time we spent with the users by attending various meetings as observers. We also simply spent time in one of the users' areas to observe the relationships between the individuals.

\subsection{SEAM}

SEAM [11, 12], is an Enterprise Architecture method. It is mainly used for business and IT alignment projects. One of the SEAM modules enables to create models of 
networks of stakeholders and their motivations in terms of goals and beliefs [8]. These models are used for the definition of early (or business) requirements. During the project we used only this goal-belief module.

The first contribution of the goal-belief models was to analyse the stakeholders' relationships. We oriented the first round of interviews so that we could clearly understand the relationships between the different actors.

The analysis was then modified to be more goal-oriented. In effect, Adoption Methodology doesn't really emphasize this aspect. The goal-belief models and goal oriented requirements engineering in general influenced this aspect of our approach.

To be precise, we used a hybrid version of the goal-belief discovery process. Instead of identifying the maintenance goals of the organisation and individuals at the beginning of the analysis, we first considered the performance goals of individuals. The performance goals of an individual are all the elements on which the individuals are evaluated. The salary and the career of the individual will depend on achieving those goals. The approach was a bottom-up individual to organisation analysis.

Concretely, we focused the interviews on the goals, belief and interests of the users. In order to optimise the limited time we had, we took into account for the first round of interviews the double challenge of asking enough why questions but not too many. It helped us to improve our analysis and understanding of the business processes and current practices.

The performance goals of every individual are influenced by the maintenance goals of the organisation. However, in the case of this project, it was more realistic to identify the performance goals of each individual and then understand which maintenance goal was influencing them rather than the other way around.

In conclusion, the two approaches have the same objective. The bottom-up is more realistic in a Procter \& Gamble context and has the important advantage that it allows focusing directly on the target

The goal-belief analysis helped us to narrow our focus on the motivations of the users. For example, it raised the importance of understanding the external factors that impact the work of the users. The organisation we considered in the analysis is not directly connected to the external world, but the external influences are represented via different ways. We modified our approach to identify those factors and tried to understand their impact on the users' goals.

\section{Academic Results of the Project}

Even though this was an industrial project, the relationship between academia and industry (i.e. between P\&G and EPFL) resulted in cross-pollination of methods. P\&G's Adoption Methodology reminded us of Moore's Chasm theory [7] on which it is based. Moore's theory is based on the well-known technology adoption lifecycle but argues that there is a chasm between the different segments represented in this cycle, i.e. innovators, early adopters, early majority, late majority and laggards.

We integrated Moore's Chasm theory into our experiential Enterprise Service Oriented Architecture (ESOA) course [10]. The course's main goals are to give undergraduate students a sense of the real problems faced by organizations and to provide them with conceptual tools to provide solutions to these problems. To make the course as real as possible we use an experiential pedagogy [6]. 
The course illustrates Business/IT alignment by showing the direct relationship between marketing and sales issues on the one hand and IT solutions on the other hand. SEAM is used throughout the course for helping with business/IT alignment.

We place the students in a situation where they must identify the problems a commercial company has in selling a revolutionary product in a conservative market. Following the P\&G project we integrated Moore's Chasm theory into the course to show the students how difficult it is to prevail over the long term in a conservative market when introducing a disruptive technology.

Just like the P\&G project, the ESOA course shows students the importance of Contextual Inquiry by placing them in a situation where traditional interviews do not permit to identify the whole set of problems faced by the stakeholders. The results of the interviews and contextual inquiry are formalized in SEAM goal-belief models.

\section{Key Learning Points}

In this section we describe the main points that we believe made this project successful and that can serve other Business/IT projects in industry.

\section{Business/IT Alignment Enables IT to Understand the Business}

On of the main principles of the project was for IDS to understand the business rather than requiring the business to understand IDS. The tools used, Adoption Methodology, Contextual Inquiry and SEAM were used for and to the extent that they helped with this principle.

\section{The Emergence of Units Such as IDS Makes RE Visible in Large Corporations}

The shift in the mission of IDS from the specification of IT systems to a focus on providing value to its (internal) clients meant that IDS had to implement RE methods. This in turn made RE visible to both clients and management. Note that we have seen this trend in another large corporation as well.

\section{The Adoption of RE Methods in Industry Requires Industry Tuned Methods}

The first element to recall and it was the main driver of our methodology choices is that the Adoption Methodology is a recommended standard by Procter \& Gamble.

The employees of a company are used to principles and not theory. They don't have enough time to understand a theory, but they can apply principles. To be used in industry, RE methods need to be tailored to the specific needs of each organization so that clear principles can be given. The key benefits of principles are that they are easy to communicate and implement.

\section{Combining Industrial and Academic Methods}

The combination of Adoption Methodology with Contextual Inquiry and SEAM is an illustration of collaboration between industry and academy. Adoption Methodology captures requirements from a management standpoint.

Adoption methodology addresses business and user benefits (strategic and operational issues). SEAM was used to analyze relationships and goals. Contextual inquiry was used to understand current practice. 
Adoption Methodology specifies concrete principles such as the use of segmentation and scenarios. It doesn't provide a theoretical basis. Conversely, SEAM is grounded in theory but provides guidelines that are more difficult to apply. By using the principles of Adoption Methodology with a mindset inspired by SEAM we were able to define better requirements than was possible with the use of just one of these methods.

\section{Requirements Engineering can become a Strategic Tool for Enterprises}

This is very important for the requirements gathering area and for the IT system development in general, because sometimes those domains lack senior management support. This approach can really stimulate their interest and make them realise the potential impact of an IT system.

It is important to approach senior management with concrete proposals of requirements that can have a strategic impact rather than waiting for them to identify those opportunities.

At the end of the project, senior management was interacting proactively with IDS to discuss those aspects. Their interest and involvement changed dramatically between the start and the end of the discovery phase. This is mainly due to the fact that they realised the possibilities of influencing employees' current practices.

\section{Internships are a Good way to Cross Pollinate between Industry and Academia} Internships represent a lightweight collaboration between industry and academia. When a student works as an intern for a Master's project, he or she has two clients to satisfy (the industrial supervisor and the professor). The academic requirements on a Master's thesis result in methods coming from research being applied to the industrial case. The industrial case, in turn, serves as a validation for the methods coming from research. This is a good way for industry and research to learn from each other and innovate, each one in its own field. In the case of the P\&G project, $P \& G$ was able to apply new requirements techniques in concert with Adoption Methodology whereas EPFL was able to improve SEAM.

\section{Conclusions}

We believe there will be a steadily growing need for Business/IT alignment methods in the industry as IT departments of large organizations are shifting their focus away from technology and operations towards tangible business impact, in essence striving to improve their business/IT alignment. Business/IT alignment methods in general and RE methods in particular represent, in theory, a perfect fit for this new kind of industrial IT.

Today's research based methods seem difficult to implement in business organizations due to their high level of abstract complexity and the in-depth knowledge required for their implementation. Both elements do not support industrial IT departments in their key task, namely to interface with the business users in the users own "language." Hence, the industry is forced to create a set of different methods with clear and simple guidelines and easy end user communication tools. To address this need for Business/IT methods in industry, we recommend specific research geared 
towards the creation of industry-oriented methods. In this research we need to analyze the strengths and weaknesses of industry developed methods. This will enable blending existing research methods with industry methods so that the products are simple and quickly usable by the industry.

\section{References}

1. Beyer, H., Holtzblatt, K.: Contextual Design: A Customer-Centered Approach to System Designs. Morgan Kaufmann, San Francisco (1997)

2. Chan, Y.E.: Why Haven't We Mastered Alignment? The Importance of the Informal Organization Structure. MIS Quarterly Executive 1(2) (2002)

3. Chan, Y.E., Reich, B.H.: IT alignment: what have we learned? Information Technology 22, 297-315 (2007)

4. Holtzblatt, K., Jones, S.: Contextual Inquiry: A ParticipativeTechnique for System Design. In: Schuler, D., Namioka, A. (eds.) Participatory Design: Principles and Practices. Lawrence Erlbaum, Mahwah (1993)

5. Luftman, J.: Key Issues for IT Executives. MIS Quarterly Executive 3(2) (2004)

6. Kolb, D.A.: Experiential learning: experience as the sourceof learning and development. Prentice Hall, Englewood Cliffs (1984)

7. Moore, G.A.: Crossing the Chasm: Marketing and Selling High-Tech Products to Mainstream Customers. Harper Business, NY (1999)

8. Regev, G., Wegmann, A.: Defining Early IT System Requirements with Regulation Principles: The Lightswitch Approach. In: 12th IEEE International Requirements Engineering Conference (RE 2004), Kyoto, Japan (2004)

9. Regev, G., Favre, J., Hayek, E., Wilson, P., Wegmann, A.: Emergence of RE in Large Corporations: Lessons Learned From an RE Project at P\&G. Technical Report, EPFL (2006)

10. Regev, G., Gause, D.C., Wegmann, A.: Experiential learning approach for requirements engineering education. Requirements Engineering 14(4), 269-287 (2009)

11. Wegmann, A.: On the Systemic Enterprise Architecture Methodology (SEAM). In: International Conference on Enterprise Information Systems (ICEIS), Angers, France (2003)

12. Wegmann, A., Regev, G., Rychkova, I., Lê, L.-S., De La Cruz, J.D., Julia, P.: Business-IT Alignment with SEAM for Enterprise Architecture. In: 11th IEEE International EDOC Conference. IEEE Press, Los Alamitos (2007) 